\title{
Preparation of chitosan/gum Arabic nanoparticles and their use as novel stabilizers in oil/water Pickering emulsions
}

\author{
Asma Sharkawy $^{\mathrm{a}}$, Maria Filomena Barreiro ${ }^{\mathrm{b}, \mathrm{c}, *}$, Alírio Egídio Rodrigues ${ }^{\mathrm{a}, * *}$ \\ ${ }^{a}$ Laboratory of Separation and Reaction Engineering - Laboratory of Catalysis and Materials (LSRE-LCM), Faculdade de Engenharia, Universidade do Porto, Rua Dr. \\ Roberto Frias $s / n$, 4200-465, Porto, Portugal \\ ${ }^{\mathrm{b}}$ Laboratory of Separation and Reaction Engineering - Laboratory of Catalysis and Materials (LSRE-LCM), Polytechnic Institute of Bragança, Campus Santa Apolónia, \\ 5301-253, Bragança, Portugal \\ ${ }^{\mathrm{c}}$ Centro de Investigação de Montanha (CIMO), Instituto Politécnico de Bragança, Campus de Santa Apolónia, 5300-253 Bragança, Portugal
}

\section{A R T I C L E I N F O}

\section{Keywords:}

Pickering emulsions

Surfactant-free emulsions

Chitosan/gum Arabic nanoparticles

Nanoparticles wettability

Rheological properties

\begin{abstract}
A B S T R A C T
Recently, there has been a renewed interest in Pickering emulsions owing to their surfactant-free nature, and the use of natural-based particles as stabilizers became a priority due to the applications they can enable. In this work, chitosan/gum Arabic (CH/GA) nanoparticles were synthesized and tested as novel stabilizers. Among the tested $\mathrm{CH} / \mathrm{GA}$ weight ratios, the particles prepared using 1:1 ratio exhibited near-neutral wettability, an average size of $108.6 \mathrm{~nm}$ and a zeta potential of $56.3 \mathrm{mv}$. Pickering emulsions prepared from these particle dispersions $(1.5 \% \mathrm{w} / \mathrm{v})$, and high oil volume fractions $(\varphi=0.6,0.7)$, have shown high storage stability. Moreover, confocal laser scanning microscopy confirmed the $\mathrm{o} / \mathrm{w}$ type and the effective adsorption of the nanoparticles at the oil/ water interface forming a barrier against droplets coalescence. The emulsions have shown shear-thinning and elastic-like behavior. These findings open new avenues for using these emulsions as novel delivery systems, e.g. in cosmetic and food applications.
\end{abstract}

\section{Introduction}

Emulsion-based systems are widely used as food and cosmetic products, and pharmaceutical dosage forms owing to their facile formulation and enhancement of active agents and drug solubilization (Harman, Patel, Guldin, \& Davies, 2019). These systems are typically stabilized by emulsifiers, which are being increasingly associated with environmental and health concerns in the long term. Alternatively, emulsions stabilized by solid particles, also known as Pickering emulsions, are emerging as promising substitutes in the areas of food, cosmetics and pharmaceuticals (Chevalier \& Bolzinger, 2013; Dickinson, 2017). They are named after S.U. Pickering who reported them in 1907 (Pickering, 1907); however, they gained relevance only recently because of the rising environmental public awareness, and also due to the advancements in the development and characterization of nanoparticles. The surfactant-free nature of Pickering emulsions makes them an excellent choice to overcome the adverse effects exhibited by the chemically synthesized emulsifiers used to prepare conventional emulsions (Chevalier \& Bolzinger, 2013).

Until the past few years, most of the research conducted on Pickering emulsions focused on the use of inorganic particles, such as silica and clay, as stabilizers (Lam, Velikov, \& Velev, 2014). However, at present, the development of organic and biopolymeric particles as Pickering stabilizers has become an important field of research; in order to achieve both biodegradability and biocompatibility with the aim of utilizing them in food, cosmetic and pharmaceutical applications (Lam et al., 2014; Laredj-Bourezg, Bolzinger, Pelletier, \& Chevalier, 2017; Marto et al., 2019). Nevertheless, production of stable Pickering emulsions based on biopolymers is relatively challenging; since polysaccharides and some proteins are naturally very hydrophilic hindering the achievement of an intermediate particle wettability and a suitable contact angle at the oil/water interface (Xiao, Wang, Perez Gonzalez, \& Huang, 2016). In this context, recent studies have successfully employed cellulose nanocrystals (Errezma, Mabrouk, Magnin, Dufresne, \& Boufi, 2018), chitin nanocrystals (Perrin, Bizot, Cathala, \& Capron, 2014) and chitin nanofibers (Larbi et al., 2018) as Pickering stabilizers.

\footnotetext{
* Corresponding author at: Laboratory of Separation and Reaction Engineering - Laboratory of Catalysis and Materials (LSRE-LCM), Polytechnic Institute of Bragança, Campus Santa Apolónia, 5301-253, Bragança, Portugal.

** Corresponding author at: Laboratory of Separation and Reaction Engineering - Laboratory of Catalysis and Materials (LSRE-LCM), Faculdade de Engenharia, Universidade do Porto, Rua Dr. Roberto Frias s/n, 4200-465, Porto, Portugal.

E-mail addresses: barreiro@ipb.pt (M.F. Barreiro), arodrig@fe.up.pt (A.E. Rodrigues).
} 
Chitosan is a linear polysaccharide, obtained by the deacetylation of chitin, a natural abundant polymer present in the exoskeleton of crustaceans. Chitosan has antibacterial, antifungal and gelling properties. It has been known as a promising material in the areas of tissue engineering, drug delivery systems, food and cosmetic applications due to its non-toxicity and biodegradability (Joye \& McClements, 2014; Muxika, Etxabide, Uranga, Guerrero, \& de la Caba, 2017). Chitosan possesses amino groups in its polymeric backbone, making its solubility pH-dependent. At low $\mathrm{pH}(<6-6.5)$, the amino groups become protonated and acquire positive charges, rendering chitosan soluble. At higher $\mathrm{pH}$ values, they are deprotonated, making chitosan uncharged and able to aggregate and precipitate. Chitosan nanoparticles have been used extensively as drug delivery systems, but recently greater attention was given to the use of these particles as Pickering emulsion stabilizers (Asfour, Elmotasem, Mostafa, \& Salama, 2017; Mwangi, Ho, Tey, \& Chan, 2016). Being a highly hydrophilic biopolymer, chitosan requires surface modification, or complexation with other polymers, to modify its wettability, and consequently its emulsification properties (Atarian, Rajaei, Tabatabaei, Mohsenifar, \& Bodaghi, 2019; Elsabee, Morsi, \& AlSabagh, 2009). Complexation of chitosan with other biopolymers is considered a facile alternative to the currently used chemical surface modification strategies.

Gum Arabic (GA), also called Acacia gum, is a dried exudate obtained from the stems and trunk of Acacia tree. It is an amphiphilic branched polysaccharide that has good emulsifying properties and low viscosity (Dickinson, 2003). It is used in different applications, such as food, pharmaceutical, biomedical, cosmetic and textile industries (Ali, Ziada, \& Blunden, 2009). The use of gum Arabic in the formulation of Pickering emulsions has been reported, recently, in very few studies, with focus mainly on food applications. In this context, (Dai, Sun, Wei, Mao, \& Gao, 2018) and (Li et al., 2018) produced Pickering emulsions stabilized by gum Arabic/zein protein particles. The particles were formed through electrostatic attraction between the two oppositely charged polymers and hydrogen bonding between them. In another study, (Wei \& Huang, 2019) stabilized edible Pickering emulsions using gum Arabic/ovotransferrin glycoprotein nanoparticles. However, no studies documented the complexation of gum Arabic with another polysaccharide biopolymer to produce nanoparticles with near-neutral wettability, which is a crucial prerequisite required to achieve high Pickering stabilization.

The main objective of this work is to produce chitosan/gum Arabic nanoparticles by a facile method, and subsequently utilize these novel biopolymeric nanoparticles to develop stable surfactant-free polysaccharide-based Pickering emulsions. The study also aims to investigate the effect of the oil phase volume fraction and nanoparticles concentration on the stability, microstructure and rheological properties of the developed emulsions. To the best of our knowledge, no previous work reported the formulation of Pickering emulsions using chitosan/gum Arabic nanoparticles.

\section{Materials and methods}

\subsection{Materials}

Chitosan (ChitoClear ${ }^{\circledR}$ ) with a degree of deacetylation of $96 \%$ and MW 100000-200000 Da was a gift from Primex ehf, Iceland. Gum Arabic (MW $=250,000 \mathrm{Da}$ ), olive oil (highly refined, low acidity), Nile Red and Nile Blue were purchased from Sigma Aldrich. Hydrochloric acid $(0.1 \mathrm{~N})$ and isopropyl alcohol were obtained from Merck, and acetic acid $(0.1 \mathrm{~N})$ was purchased from Alfa Aesar.

\subsection{Chitosan/gum Arabic ( $C H / G A)$ nanoparticles}

\subsubsection{Synthesis procedure}

The nanoparticles were prepared following the method of (Tan, Xie, Zhang, Cai, \& Xia, 2016) with slight modifications; namely the process was applied without the addition of a hydrophobic core or emulsifier. Nanoparticles of different CH/GA weight ratios (4:1, 2:1, 1:1, 1:2 and $1: 4)$ were prepared at a fixed concentration of $1.5 \%(\mathrm{w} / \mathrm{v})$. Briefly, and considering the example of CH/GA 1:1, chitosan was dissolved in $0.1 \mathrm{~N}$ acetic acid at a concentration of $1.5 \% \mathrm{w} / \mathrm{v}(0.75 \mathrm{~g}$ chitosan in $50 \mathrm{ml}$ of acetic acid), followed by the dropwise addition of $50 \mathrm{ml}$ of gum Arabic solution of the same concentration. The mixture was kept under constant magnetic stirring ( $800 \mathrm{rpm}$ ) during $30 \mathrm{~min}$ to allow complexation between chitosan and gum Arabic. An opalescent dispersion was obtained indicating a successful formation of the particles. The $\mathrm{pH}$ of the final dispersion was 4 .

\subsubsection{Wettability measurements}

The wettability of the produced CH/GA particles, conducted using an optical contact angle measuring device (OCA15 Plus, Dataphysics, Germany), was determined to evaluate their hydrophilic/hydrophobic character. The prepared $\mathrm{CH} / \mathrm{GA}$ nanoparticles dispersions were placed on smooth glass slides separately and allowed to dry at room temperature. The dried film was placed on the device platform, and then $4 \mu \mathrm{l}$ of deionized water was deposited on the surface using a high-precision injector at $25^{\circ} \mathrm{C}$. Images of the formed drops were acquired using a digital camera coupled to the equipment and the measurements were averaged over at least 4 drops. The contact angle values were calculated automatically by the equipment software by fitting the profile data of the formed droplets to the Laplace-Young equation.

\subsubsection{Transmission electron microscopy (TEM)}

The morphology of the particles was examined by TEM. Briefly, $10 \mu \mathrm{l}$ of the freshly prepared nanoparticles dispersion was diluted $(1 \mathrm{ml}$ of the nanoparticles dispersion was diluted to $10 \mathrm{ml}$ using deionized water) and mounted on Formvar/carbon film-coated mesh nickel grids (Electron Microscopy Sciences, Hatfield, PA, USA) and left standing for $2 \mathrm{~min}$. The liquid in excess was removed with filter paper. Visualization was carried out by a JEOL JEM 1400 TEM at $120 \mathrm{kV}$ (Tokyo, Japan). Images were digitally recorded using a CCD digital camera Orious $1100 \mathrm{~W}$ (Tokyo, Japan).

\subsubsection{Particle size and zeta potential}

The average diameter, zeta potential and polydispersity index (PDI) were determined by a dynamic light scattering particle size analyzer (ZetaSizer Nano ZS ZEN3600 from Malvern Instrument). For that, the samples were diluted to an appropriate concentration (100-fold serial dilution) with deionized water. All measurements were carried out at $25^{\circ} \mathrm{C}$, and the results were reported as averages of three consecutive readings.

\subsection{Pickering emulsions}

\subsubsection{Preparation procedure}

To prepare the oil/water Pickering emulsions from the CH/GA nanoparticles, olive oil was slowly added to the nanoparticles dispersion with continuous stirring at 13,500 rpm for $7 \mathrm{~min}$, using an Ultraturrax DI 25 basic IKA homogenizer. The total volume of the emulsion was fixed to $100 \mathrm{ml}$. To study the effect of the oil volume fraction on emulsion properties, emulsions of different oil volume fractions $(\varphi=0.3,0.4,0.5,0.6,0.7$ and 0.8 ) were prepared from $\mathrm{CH} / \mathrm{GA} 1: 1$ nanoparticles at a fixed concentration of $1.5 \%(\mathrm{w} / \mathrm{v})$. To study the effect of nanoparticles concentration, emulsions were prepared using dispersions with different concentrations $(0.5 \%, 1 \%, 1.5 \%$ and $2 \% \mathrm{w} / \mathrm{v})$ of $\mathrm{CH} / \mathrm{GA}$ 1:1 nanoparticles (synthesized following the procedure described in Section 2.2.1), and a fixed oil volume fraction of $\varphi=0.6$.

\subsubsection{Microstructure of Pickering emulsions}

The morphology of the formed emulsions was examined by optical microscopy using a Leica DM 2000 optical microscope equipped with a Leica Application Suite Interactive Measurement imaging software. 


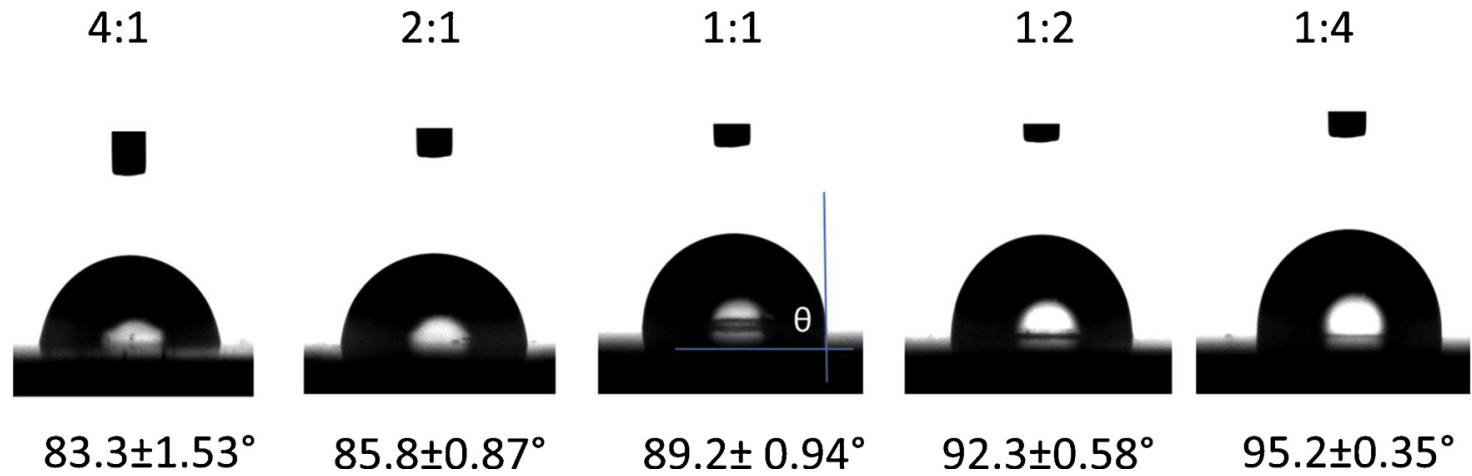

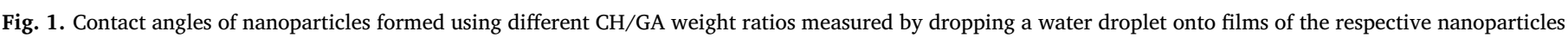
in air environment.

Moreover, confocal laser scanning microscopy (CLSM) was used to examine the interfacial microstructure, and to visualize the location of $\mathrm{CH} / \mathrm{GA}$ nanoparticles. These assays were conducted using a Leica TCS SP5 CLSM (Leica Microsystems Inc., Germany). Briefly, after their preparation, the emulsions were stained with a mixture of fluorescent dyes in isopropyl alcohol consisting of Nile red and Nile blue $(0.1 \% \mathrm{w} / \mathrm{v}$ each). Nile red was used to stain the oil phase and Nile blue for staining the nanoparticles. The CLSM was operated using two laser excitation wavelengths; $488 \mathrm{~nm}$ for the Nile red and $633 \mathrm{~nm}$ for the Nile blue.

\subsubsection{Droplet size of Pickering emulsions}

The average droplet size was measured using a laser diffraction particle size analyzer (LS 230, Beckman Coulter, USA). The refractive indexes for olive oil and water were 1.46 and 1.33 , respectively.

\subsubsection{Creaming index}

The creaming index (CI\%) determination was used as an indicator for the stability of the emulsions against separation. It was calculated according to the following equation (Li et al., 2018; Mwangi et al., 2016):

$C I(\%)=\frac{H s}{H t} \times 100$

where $\mathrm{H}_{\mathrm{s}}$ is the height of the serum layer and $\mathrm{H}_{t}$ is the total emulsion height.

\subsubsection{Rheological studies}

The rheological studies were conducted to investigate the effect of the oil volume fraction and the nanoparticles concentration on flow behavior and viscoelastic properties of the formulations. The measurements were done using a Kinexus Pro Rheometer (Malvern, UK). All the measurements were conducted at $25^{\circ} \mathrm{C}$. The steady flow measurements were performed using a cone plate of angle $4^{\circ}$ with a diameter of $40 \mathrm{~mm}$ and a fixed gap of $0.15 \mathrm{~mm}$. The emulsions were placed on the plate and allowed to stand $5 \mathrm{~min}$ for temperature equilibrium before measurements. The apparent viscosity $(\eta)$ was determined over a shear rate range from 0.1 to $100 \mathrm{~s}^{-} 1$. The frequency sweep measurements were done using a parallel plate (diameter $20 \mathrm{~mm}$ ) with a fixed gap of $1 \mathrm{~mm}$. All the dynamic tests were performed within the linear viscoelastic region. The frequency was oscillated from 0.01 to $10 \mathrm{~Hz}$ and the strain was made at $1 \%$. The elastic modulus $\left(\mathrm{G}^{\prime}\right)$ and loss modulus ( $\left.\mathrm{G}^{\prime \prime}\right)$ were recorded versus frequency.

\section{Results and discussion}

\subsection{Production and characterization of $C H / G A$ nanoparticles}

The production of $\mathrm{CH} / \mathrm{GA}$ nanoparticles was based on ionotropic gelation; which occurs due to electrostatic attraction between oppositely charged polyelectrolytes (Avadi et al., 2010). The degree of electrostatic attraction between the positively charged $\mathrm{CH}$ and the negatively charged GA is dependent on the $\mathrm{pH}$ and also on the used $\mathrm{CH} /$ GA weight ratio (Butstraen \& Salaün, 2014). It has been reported that the coacervate yield of $\mathrm{CH}$ and GA is maximized at $\mathrm{pH} 3.5-5$, and that at $\mathrm{pH} 4$ the two polymers form homogenous particles (Tan et al., 2016). Hence, this $\mathrm{pH}$ was used in the present work.

\subsubsection{Wettability of nanoparticles}

An important prerequisite for particles to be used as Pickering stabilizers is that they should be partially wetted by both the dispersed and the continuous phase in order to achieve the required emulsion stabilization. The particles wettability reflects their degree of hydrophilicity/hydrophobicity, similarly to how the hydrophilic-lipophilic balance (HLB) scale is used to describe the amphiphilic properties of the conventional surfactants (Xiao, Li, \& Huang, 2016). To assess this behavior, the contact angle $(\theta)$ is used to quantify the surface wettability of the solid particles. The value of the contact angle determines the type of the formed Pickering emulsion. Thus, hydrophilic particles have low contact angle $\left(<90^{\circ}\right)$, and stabilize preferentially $\mathrm{o} / \mathrm{w}$ emulsions, whereas hydrophobic particles form w/o emulsions and have high values of contact angle $\left(>90^{\circ}\right)$ (Dickinson, 2017). The maximum adsorption energy of a particle at the oil-water interface is achieved when the contact angle equals $90^{\circ}$, which contributes to maximizing emulsion stability as the particles will be strongly adsorbed at the oil/water interface forming a layer that sterically hinders the coalescence of the emulsion droplets (Binks \& Clint, 2002).

Fig. 1 shows the measured contact angle for the nanoparticles prepared with different $\mathrm{CH} / \mathrm{GA}$ weight ratios (4:1, 2:1, 1:1, 1:2 and 1:4). It was observed that the contact angle increases as the amount of gum Arabic in the nanoparticles increases. This behavior could be attributed to the amphiphilic moieties present in the structure of gum Arabic, namely arabinogalactan-protein fraction (AGP), which contains both hydrophobic polypeptide chains and hydrophilic polysaccharide blocks which provide it with excellent interfacial properties (Hu et al., 2019; McClements \& Gumus, 2016). On the other hand, chitosan is known to have weak surface activity due to the absence of hydrophobic moieties in its structure (Elsabee et al., 2009). It has been reported that chitosan nanoparticles have a contact angle of $42.47 \pm 1.19^{\circ}$ (Asfour et al., 2017). Thus, the results obtained in the present work suggest that GA played an important role in enhancing the wettability of the produced particles and tuning the value of the contact angle close to $90^{\circ}$. The results show that the hydrophilic character of the nanoparticles decreased with increasing the amount of GA due to its hydrophobic moieties. By analyzing the produced nanoparticles, it can be observed that the contact angle for the particles formed using a CH/GA weight ratio of $1: 1$ at $\mathrm{pH} 4$ was $89.2^{\circ}$, indicating their hydrophilic nature and thus the preferable tendency to stabilize o/w emulsions. The value of the contact angle of the CH/GA nanoparticles prepared with a weight 


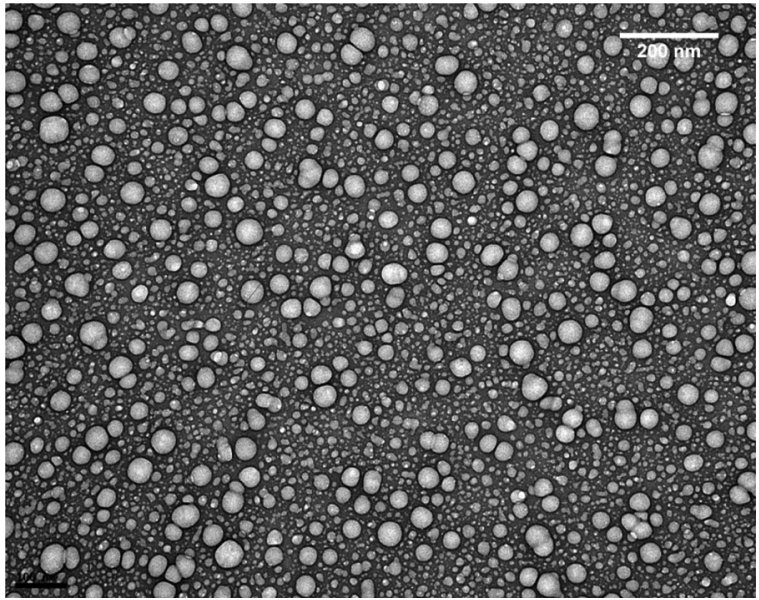

Fig. 2. TEM image of $\mathrm{CH} / \mathrm{GA} 1: 1$ nanoparticles $(1.5 \% \mathrm{w} / \mathrm{v})$, under a magnification of $100,000 \times$

ratio of $1: 1$ was the closest to $90^{\circ}$ which suggests, among the tested nanoparticles, that they have higher potential for Pickering emulsion stabilization. Therefore, in light of this conclusion, this study proceeds with the use of the particles produced with a $\mathrm{CH} / \mathrm{GA}$ weight ratio of 1:1 (CH/GA 1:1).

\subsubsection{Size, zeta potential and morphology of $C H / G A$ nanoparticles}

The freshly prepared CH/GA 1:1 nanoparticles had an average size (in number) of $108.6 \mathrm{~nm}$, a polydispersity index (PDI) of 0.218 and a zeta potential of $+56.3 \mathrm{mv}$. The measurement was repeated for the same nanoparticles dispersion after a storage period of 8 days, showing an increase in the average size to $183.07 \mathrm{~nm}$, whereas the PDI was roughly maintained $(0.221)$, pointing out only minor particles agglomeration during storage. This is attributed to the high zeta potential value of the formed nanoparticles $(>30 \mathrm{mV}$ ), which is an indication of high stability. The results are in agreement with the monodisperse nanoparticles produced at $\mathrm{pH} 4$ using $\mathrm{CH} / \mathrm{GA}$ weight ratio of $1: 1$ that had a high positive zeta potential value (Tan et al., 2016).

TEM analysis revealed that the CH/GA 1:1 nanoparticles are predominantly spherical, but also some are oval in shape (Fig. 2). The nonhomogeneity in the particles morphology could be attributed to the lack of a hydrophobic core, and hence the lack of a spherical template where the two biopolymers could deposit on. By comparing the size of the particles observed in the TEM images with the one previously determined by dynamic light scattering (DLS), it was observed that the one obtained by TEM is lower. This can be due to the fact that particles in the DLS measurements are in wet conditions (hydrated), while in the case of TEM the particles are dehydrated (Yang, Liu, \& Han, 2017).

\subsection{Production and characterization of Pickering emulsions}

\subsubsection{Effect of the oil volume fraction on morphology and stability}

To study the effect of the oil volume fraction, Pickering emulsions with different oil fractions $(\varphi=0.3,0.4,0.5,0.6,0.7$ and 0.8$)$ were produced using the nanoparticles dispersion CH/GA 1:1 nanoparticles with a fixed concentration of $(1.5 \% \mathrm{w} / \mathrm{v})$. The morphology of the produced Pickering emulsions was examined by optical microscopy, and the obtained results are shown in Fig. 3. As expected, since the preparation conditions (total emulsion volume, stirring rate and time) were the same for all samples, the emulsion droplet size increased as the oil volume fraction increased. This observation is consistent with the decreased total amount of nanoparticles available to be adsorbed at the oil/water interface, which results in the formation of droplets of larger size. These results are in agreement with the findings reported in previous studies (Atarian et al., 2019; Dai, Sun et al., 2018; Li et al.,
2018). Moreover, when the oil volume fraction increased above 0.7 ( $\varphi=0.8$ ), oiling off and droplet flocculation were noticed, and the droplets started to deform by losing their spherical shape; indicating that the number of the available nanoparticles was not enough to stabilize the Pickering droplets against coalescence. A similar observation was noticed in zein/gum Arabic Pickering emulsions with an oil fraction above 0.6 (Li et al., 2018). For this reason, this sample ( $\varphi=0.8$ ) was discharged from subsequent characterizations.

Fig. 4A shows the digital photographs of the freshly produced Pickering emulsions for the tested volume fractions in the range 0.3-0.7, and Fig. 4B shows their aspect after a two-month storage period showing the corresponding creaming index (CI\%). It can be observed that formulations with lower olive oil fractions underwent faster separation and had higher values of $\mathrm{CI} \%$, indicating poor storage stability. However, when the oil fraction increased from 0.3 to 0.5 , the value of the $\mathrm{CI} \%$ decreased from $50.7 \%$ to $17.8 \%$, implying that more stable Pickering emulsions are formed as the oil volume fraction increases. Additionally, formulations with high oil volume $(\varphi=0.6$ and 0.7 ) have shown no gravitational phase separation during the tested storage period of two months $(\mathrm{CI} \%=0)$, suggesting their high stability. Moreover, the formulation with $\varphi=0.7$ exhibited the densest/thickest appearance/texture (gel-like appearance) (Fig. 4C), as noticed by visual inspection and was confirmed further by the performed rheological studies (Section 3.2.4). The results of the evolution of the average size (obtained by laser diffraction) of the emulsion droplets as the oil volume fraction increases (Fig. 4D) were consistent with the observations made by the optical microscopy.

\subsubsection{Effect of nanoparticles concentration on morphology and stability}

To assess the effect of nanoparticles concentration, the oil volume fraction was fixed $(\varphi=0.6)$ and dispersions of CH/GA 1:1 nanoparticles with concentrations of $0.5 \%, 1 \%, 1.5 \%$ and $2 \%(\mathrm{w} / \mathrm{v})$ were used. Fig. 5 shows the optical microscopy images taken for each prepared formulation. From the observation of the images, it can be perceived that the sample prepared with the dispersion of low nanoparticles concentration $(0.5 \% \mathrm{w} / \mathrm{v})$ gave rise to a Pickering emulsion with non-uniformly spherical pattern. However, Pickering droplets consistently acquired a uniform spherical morphology at higher CH/GA nanoparticles concentrations. In addition, the average droplet size was found to decrease as the nanoparticles concentration increases. This behavior is supported by the fact that a higher $\mathrm{CH} / \mathrm{GA}$ nanoparticles concentration induces larger interfacial area coverage, limiting droplets coalescence, which results in smaller droplet sizes (higher stabilized interfacial area). These results indicate that the emulsification efficiency of the produced Pickering emulsions is directly linked to the ability of CH/GA nanoparticles to cover the oil droplets at the interface. Similar observations were noticed with chitosan (Mwangi et al., 2016) and kafirin (Xiao, Wang et al., 2016) nanoparticles-stabilized Pickering emulsions.

Concerning the storage stability of the produced emulsions, it was observed that it increased as the CH/GA 1:1 nanoparticles concentration increased. Fig. $6 \mathrm{~A}$ and $\mathrm{B}$ show that the samples prepared with nanoparticles concentrations of $1.5 \%$ and $2 \% \mathrm{w} / \mathrm{v}$ did not develop any phase separation after the studied storage period of two months. The determined $\mathrm{CI} \%$ values remained $0 \%$, indicating that the presence of higher CH/GA 1:1 nanoparticles concentration promoted a higher degree of coverage of the oil droplets, which inhibited their aggregation and subsequently prevented the creaming and separation phenomena. Furthermore, it was observed that when the nanoparticles concentration was increased, the formed Pickering emulsions exhibited thicker gel-like texture. Fig. 6C shows the formulation prepared with nanoparticles concentration of $2 \% \mathrm{w} / \mathrm{v}$ which had the densest appearance among the tested concentrations. This was further assessed and confirmed by the rheological measurements. It was also found that the emulsion droplet average size decreased as the nanoparticles concentration increased (Fig. 6D), which is in agreement with the average 

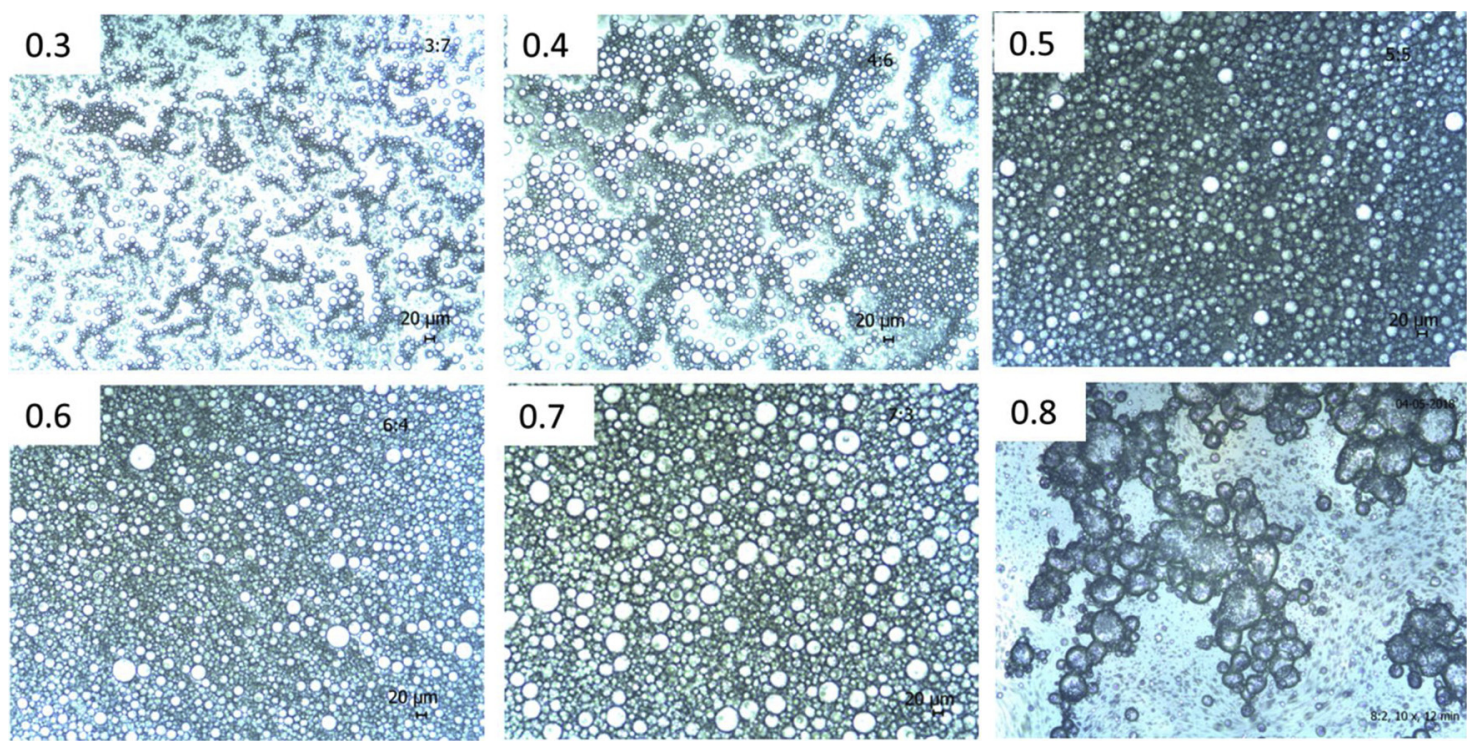

Fig. 3. Optical microscope images of Pickering emulsions prepared with different oil volume fractions and a fixed concentration of $\mathrm{CH} / \mathrm{GA}$ 1:1 nanoparticles (1.5\% $\mathrm{w} / \mathrm{v})$.
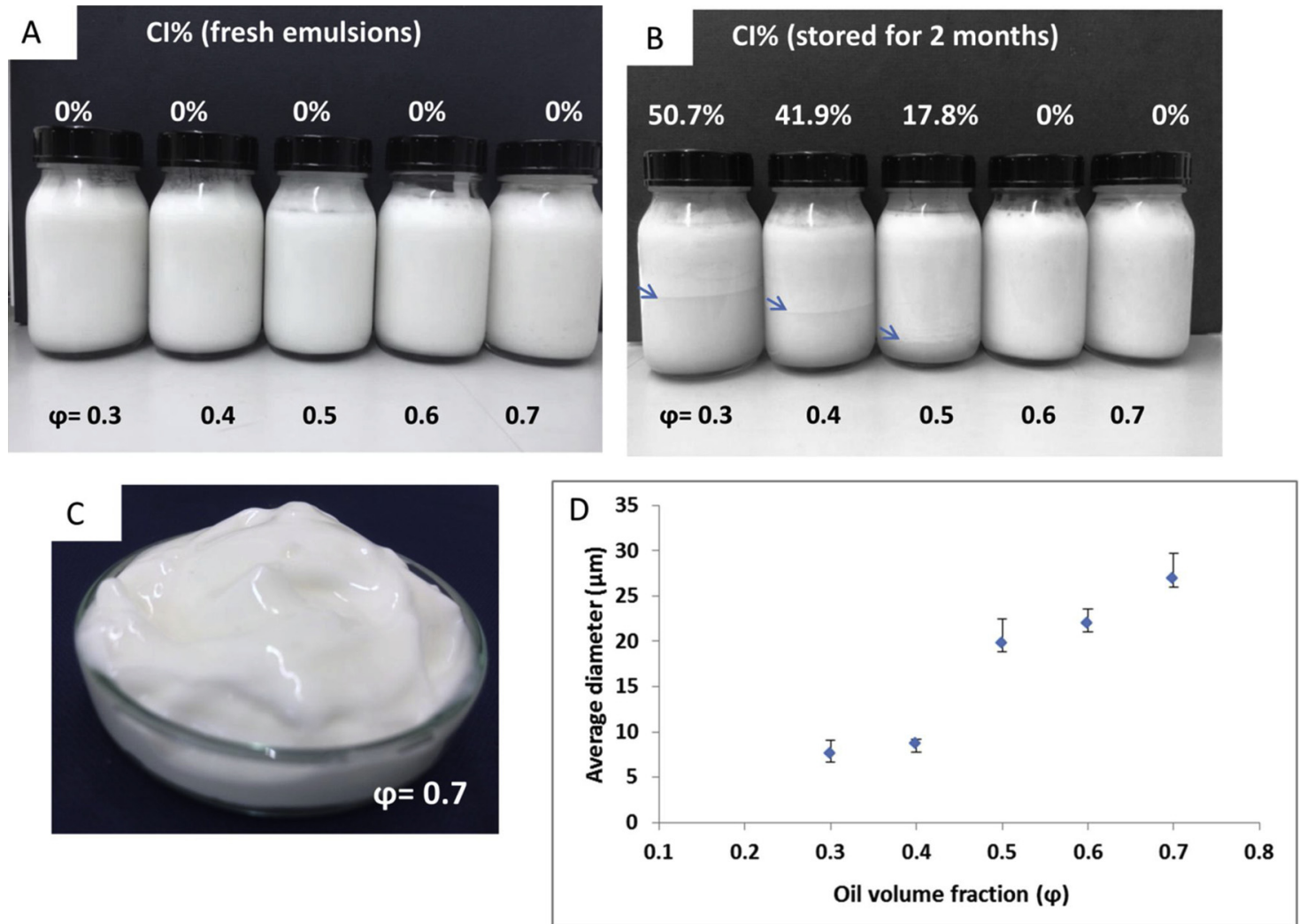

Fig. 4. Visual appearance and creaming index \% (CI\%) values of Pickering emulsions prepared with oil volume fractions of $0.3,0.4,0.5,0.6$ and 0.7 , and a dispersion of $\mathrm{CH} / \mathrm{GA} 1: 1$ nanoparticles $(1.5 \% \mathrm{w} / \mathrm{v})$ right after preparation (A); after 2 months at room temperature (the arrows point to separation if present) (B); visual appearance of the formulation with $\varphi=0.7$ (C); and average droplet size for the tested formulations (D).

sizes observed previously by the optical microscopy.

\subsubsection{Interfacial structure of Pickering emulsions}

CLSM was used to examine the interfacial structure and to confirm the morphology and type of the formed Pickering emulsions. CLSM images (Fig. 7) reveal that the nanoparticles form a distinct layer (seen as red fluorescent halos) at the boundary of the oil droplets which act as a barrier against coalescence. The images also show that the oil phase (seen as green spheres) is the dispersed phase confirming the formation of an $\mathrm{o} / \mathrm{w}$ emulsion, and that no phase inversion took place. It could be observed that when the oil volume fraction increased from 0.5 (Fig. 7A) to 0.7 (Fig. 7B), using a fixed CH/GA 1:1 nanoparticles concentration $(1.5 \% \mathrm{w} / \mathrm{v})$, the oil droplets maintained their spherical shape and increased in size. A similar observation was noticed in $\mathrm{o} / \mathrm{w}$ emulsions stabilized with zein/gum Arabic nanoparticles, which at a high oil fraction (0.7) showed no phase inversion (Dai, Sun et al., 2018). 

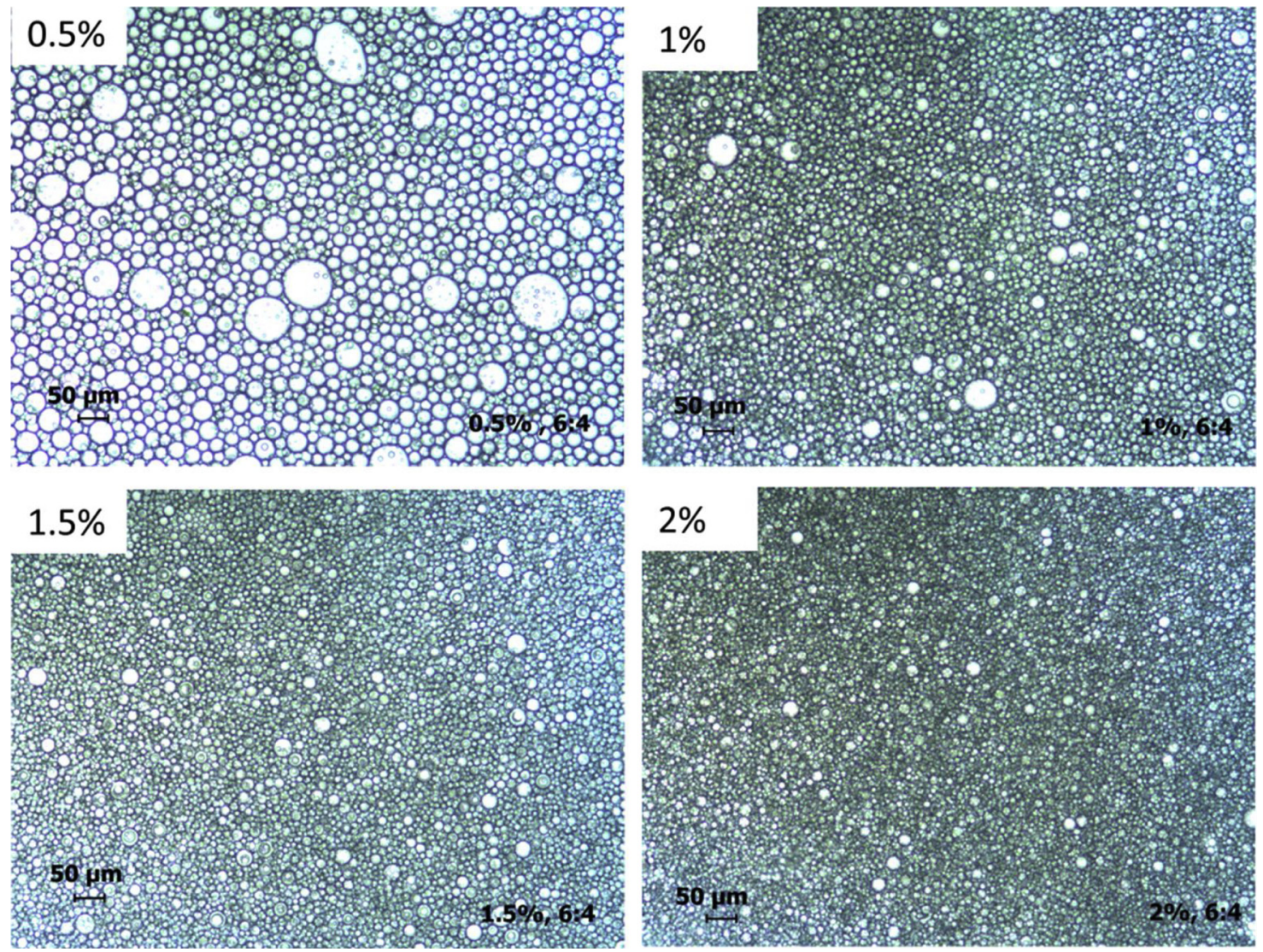

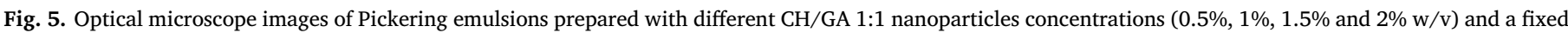
oil volume fraction $(\varphi=0.6)$.

However, deformation and merging of emulsion droplets were observed in emulsions stabilized with chitosan/zein nanoparticles when a similar oil volume was used (Wang et al., 2016).

\subsubsection{Rheology of Pickering emulsions}

The rheological properties of the emulsions are significantly linked to their properties and performance, as well as their storage stability (Liu et al., 2019). The effect of the oil volume fraction on the rheological properties of the prepared Pickering emulsions was studied considering the formulations prepared with $\varphi=0.5,0.6$ and 0.7 , and using $\mathrm{CH} / \mathrm{GA} 1: 1$ nanoparticles dispersion with a concentration of $1.5 \%$ (w/ v). Fig. 8A shows that, for the three tested formulations, the apparent viscosity decreased as the shear increased from 0.1 to $100 \mathrm{~s}^{-1}$, indicating a shear-thinning and a non-Newtonian fluid behavior. The reduction in viscosity reflects the deflocculation of the emulsion droplets and the breaking of the internal structure upon increasing the shear rate (Dai, Sun et al., 2018; Li et al., 2018). In addition, the formulations have shown an increase in viscosity as the oil volume fraction increased. These results are in agreement with previous reports on the rheological properties of Pickering emulsions (Dai, Sun et al., 2018; Li et al., 2018; Xiao, Wang et al., 2016).

Frequency sweep measurements were done to determine the storage modulus $\left(G^{\prime}\right)$ and the loss modulus $\left(G^{\prime \prime}\right)$ versus frequency increase. The storage modulus reflects the elastic solid-like character of the material, whereas the loss modulus indicates the viscous response and measures the dissipated energy. It was observed that $G^{\prime}$ and $G^{\prime \prime}$ values increased as the oil fraction increased (Fig. 8B). Additionally, it was observed for an oil volume fraction of $0.5, \mathrm{G}^{\prime \prime}$ was slightly higher than $\mathrm{G}^{\prime}$; indicating the liquid nature of the emulsion, whereas for the formulations with high oil volume fraction (0.6 and 0.7), it was observed that $\mathrm{G}^{\prime}>\mathrm{G}^{\prime \prime}$. This reflects that the rheological behavior of formulations with higher oil volume fraction is predominantly elastic, i.e. they are composed of elastic gel solid-like networks. Fig. 8B also shows that the values of $\mathrm{G}^{\prime}$ and $G^{\prime \prime}$ rose with oil volume fraction increase. Recently published studies also reported similar behavior for $\mathrm{G}^{\prime}$ and $\mathrm{G}^{\prime \prime}$ as the oil volume fraction in Pickering emulsions increases (Dai, Sun et al., 2018; Dai, Zhan et al., 2018; Wang et al., 2016). These results can be explained by the fact that the oil droplets in highly concentrated emulsions become more densely packed, resulting in high $\mathrm{G}^{\prime}$ values and high resistance to deformation by shear stress. It can be concluded from both assays that the oil volume fraction had a significant impact on the rheological properties of the Pickering emulsions. Furthermore, it is generally accepted that the presence of gel-like structure stabilizes the emulsion droplets by hindering their free movement, and thus prevent the occurrence of phase separation (Wei \& Huang, 2019). In addition, according to Stokes' law, gravitational separation rate is related to the system viscosity; as phase separation increases when viscosity decreases. Therefore, the higher the viscosity of the formulation, the higher is the expected stability (Li et al., 2018; Mwangi et al., 2016).

The effect of the nanoparticles concentration on the rheological properties of the produced Pickering emulsions was tested by using the samples prepared from dispersions with $1 \%, 1.5 \%$ and $2 \%(\mathrm{w} / \mathrm{v}) \mathrm{CH} /$ GA 1:1 nanoparticles, and a constant oil volume fraction of 0.6 . It can be observed from Fig. 9A that the emulsions exhibited shear-thinning behavior as their viscosity decreased with shear rate increase. It can be also inferred that the apparent viscosity of the emulsions increased as the nanoparticles concentration increases, indicating the formation of a stronger structural network. This behavior was described in studies using other Pickering stabilizers, such as kafirin (Xiao, Wang et al., 2016), ovotransferrin/gum Arabic (Wei \& Huang, 2019), and octylgrafted alginate amide gel particles (Yang et al., 2017). This can be attributed to the fact that increasing the concentration of Pickering 

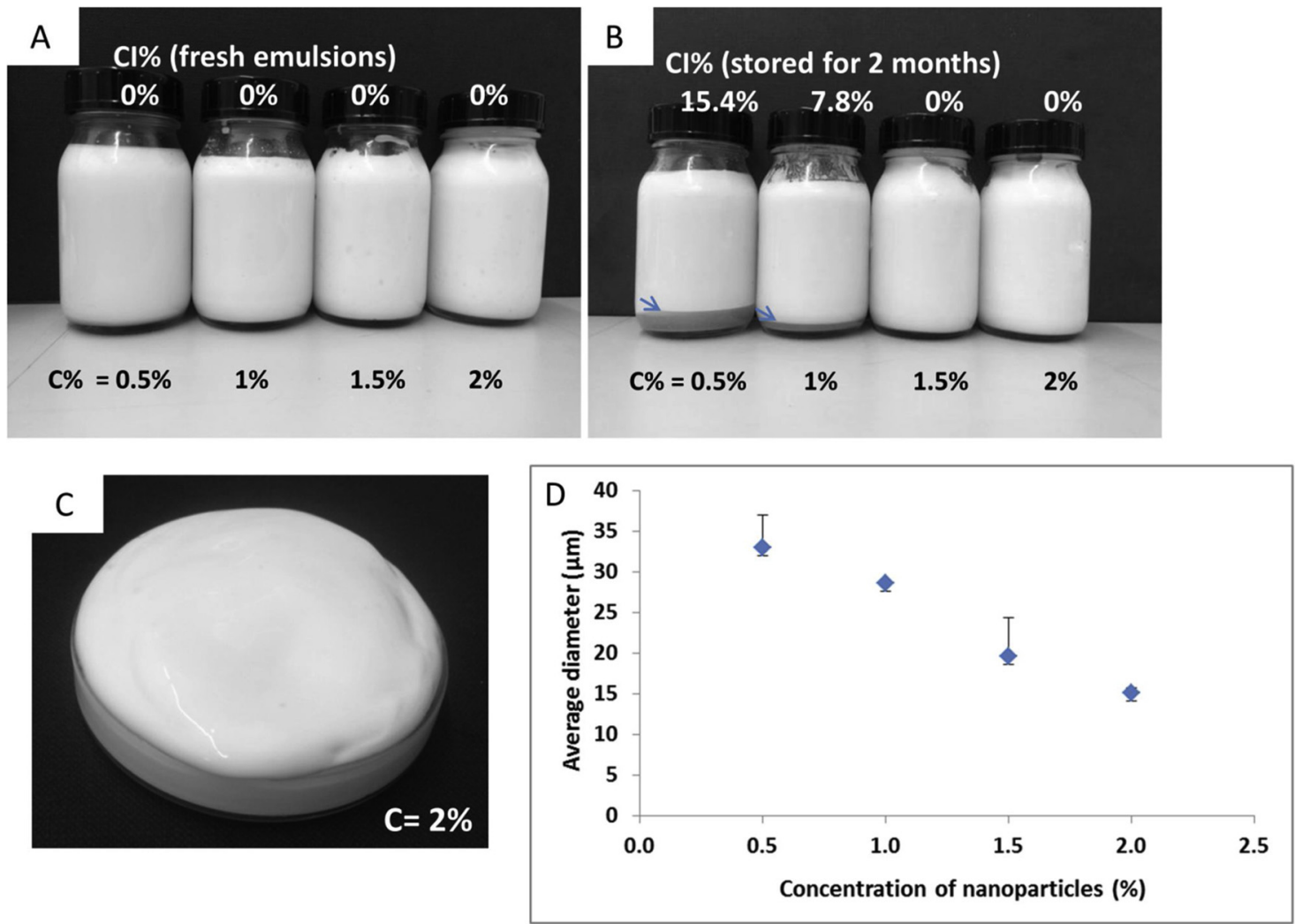

Fig. 6. Visual appearance and creaming index (CI\%) values of Pickering emulsions produced with different concentrations of $\mathrm{CH} / \mathrm{GA} 1: 1$ nanoparticles $(\mathrm{C}=0.5 \%$, $1 \%, 1.5 \%$ and $2 \% \mathrm{w} / \mathrm{v})$ using a fixed oil volume fraction $(\varphi=0.6)$ right after preparation (A); and after 2 months at room temperature (the arrows point to separation if present) (B); visual appearance of formulation with $\mathrm{C}=2 \% \mathrm{w} / \mathrm{v}(\mathrm{C})$; and average droplet size for the tested formulations (D).
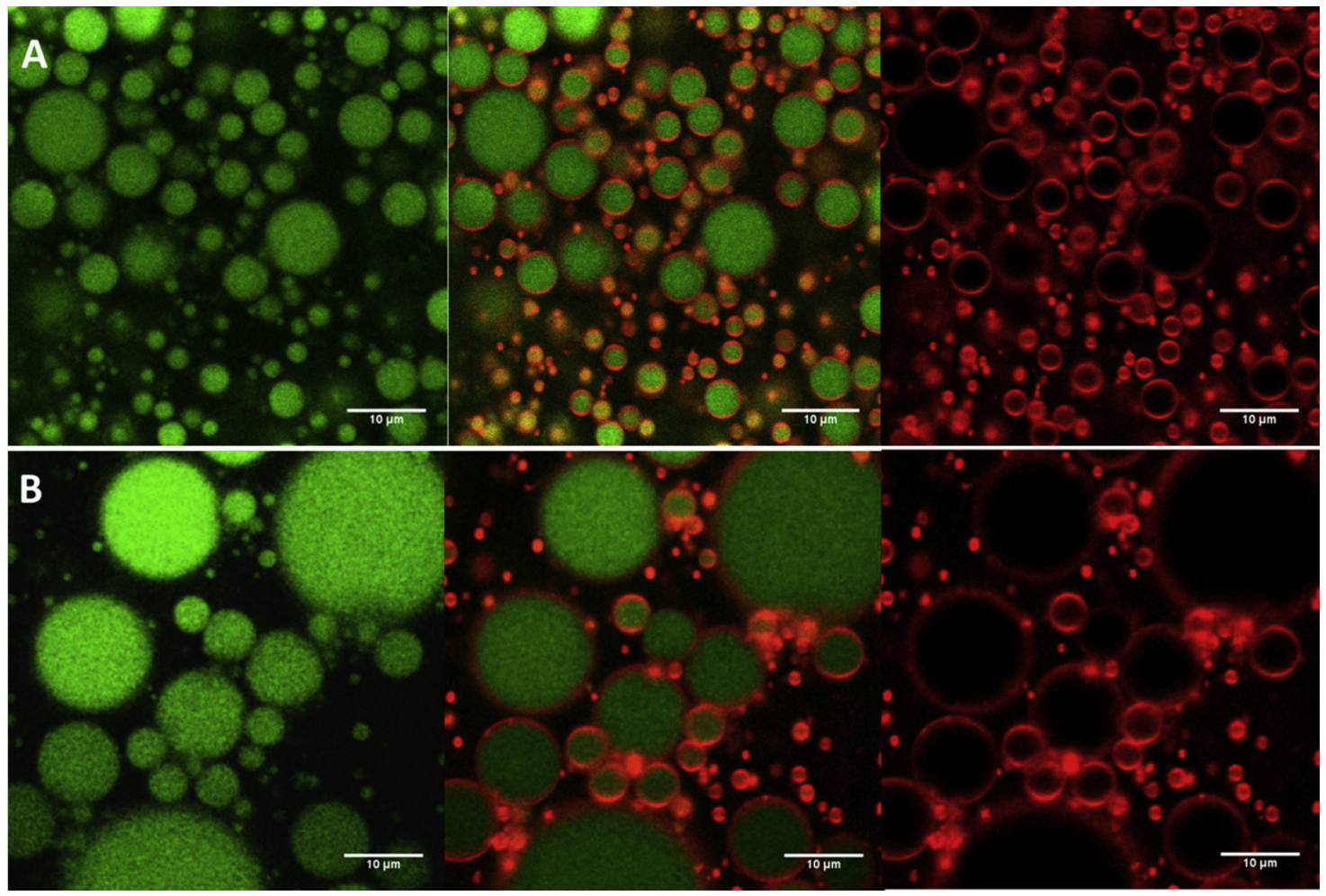

Fig. 7. Confocal laser scanning microscopy images of formulations prepared with a fixed concentration of CH/GA 1:1 nanoparticles dispersion (1.5\% w/v) and an oil volume fraction of (A) $\varphi=0.5$; and (B) $\varphi=0.7$. The emulsion oil phase appears in green (on the left), whereas the adsorbed nanoparticles appear in red (on the right). The images in the middle are an overlay of these two images (right and left) (For interpretation of the references to colour in this figure legend, the reader is referred to the web version of this article). 

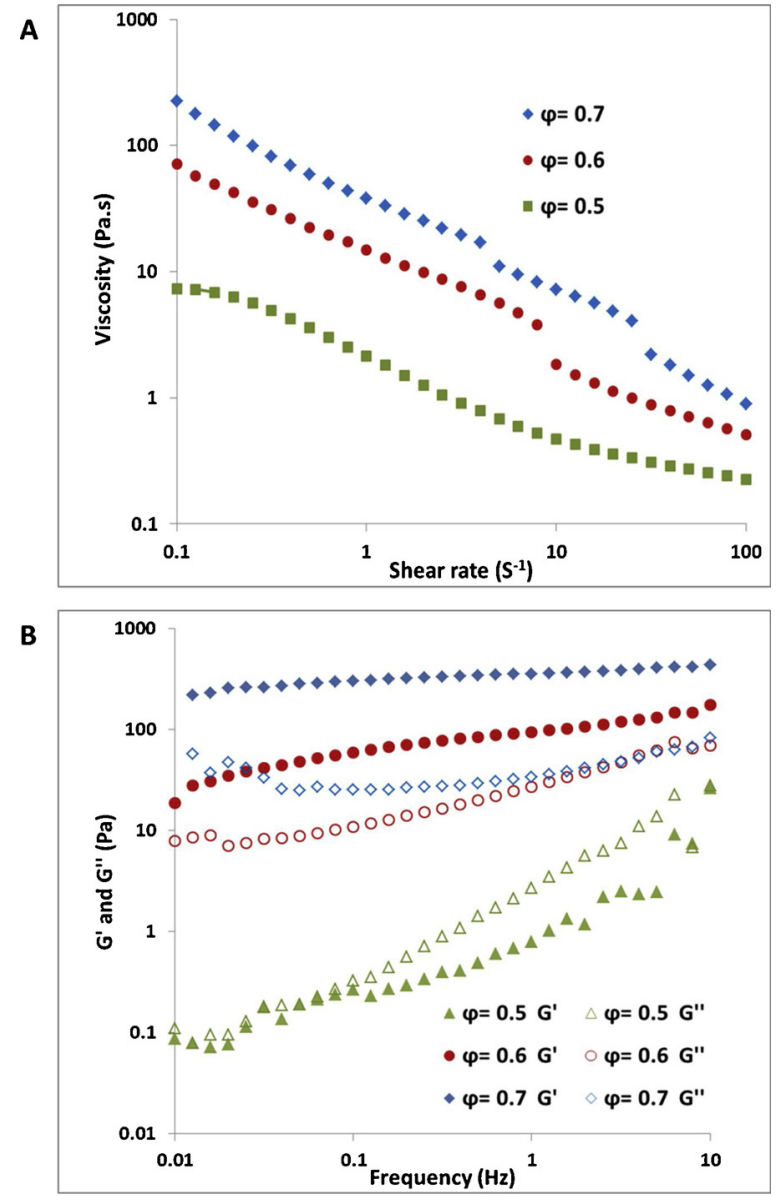

Fig. 8. Effect of oil volume fraction on rheological properties; A) Apparent viscosity versus shear rate; B) Oscillatory frequency sweep curves. G': storage modulus; and $\mathrm{G}^{\prime \prime}$ : loss modulus.

particles causes the emulsion droplets to be in closer proximity, and thus packed in a denser way. This dense distribution of emulsion droplets results in higher viscosity and makes them more resistant to deformation (Wei \& Huang, 2019). Furthermore, all the formulations have shown higher $\mathrm{G}^{\prime}$ values in comparison with $\mathrm{G}^{\prime \prime}$ (Fig. 9B), which indicates that they have predominantly elastic properties, namely due to the formation of elastic gel networks between the nanoparticles and oil droplets. Taken together, these findings show that the rheology of Pickering emulsions stabilized by $\mathrm{CH} / \mathrm{GA}$ nanoparticles can be tuned by altering the composition of the emulsion; by either changing the oil volume fraction or the nanoparticles concentration.

\section{Conclusions}

In this work, $\mathrm{CH} / \mathrm{GA}$ nanoparticles have been synthesized and their use as novel stabilizers for Pickering emulsions was tested. This strategy ensures the production of surfactant-free emulsions, an interesting topic in the context of sustainable and green chemistry principles. GA with its hydrophobic moieties had an important function in adjusting the wettability of the developed particles. The nanoparticles produced using $\mathrm{CH} / \mathrm{GA}$ weight ratio of 1:1 demonstrated near-neutral wettability (contact angle close to $90^{\circ}$ ). CLSM analysis revealed that the nanoparticles formed a very perceptible layer around the oil droplets, which prevents droplets from coalescence assuring emulsion stability. Moreover, it was shown that gravitational separation on long-term storage was inhibited by increasing the oil volume fraction and the nanoparticles concentrations in the produced Pickering emulsions. The formulations were found to be stable during the tested storage period of
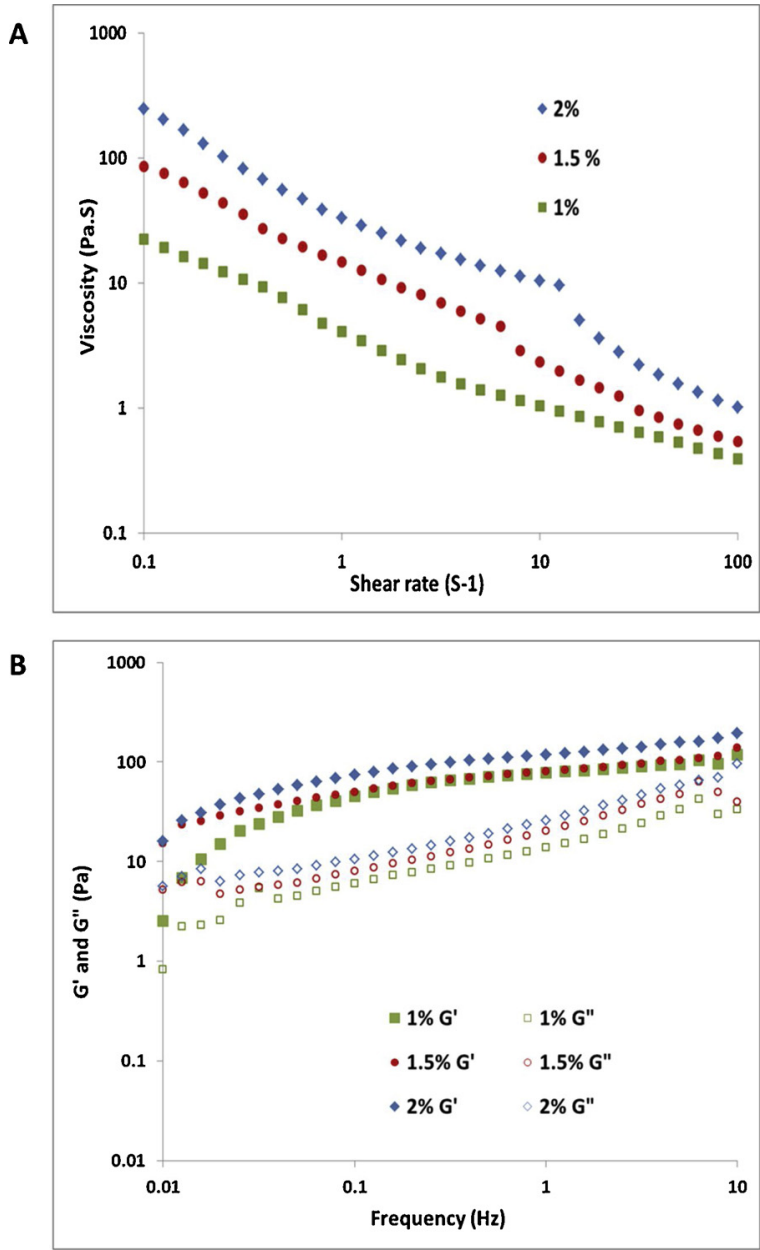

Fig. 9. Effect of the concentration of nanoparticles on rheological properties; A) Apparent viscosity versus shear rate; B) Oscillatory frequency sweep curves. G': storage modulus; and $\mathrm{G}^{\prime \prime}$ : loss modulus.

two months. The rheological properties of the formulations were shown to be tunable; as they depended on the oil volume fraction and the nanoparticles concentration in the emulsions. In addition, they have shown shear thinning and elastic-like behavior. This work provides new insights for $\mathrm{CH} / \mathrm{GA}$ based Pickering emulsions as novel surfactant-free biocompatible emulsion systems. Future efforts will explore using these Pickering emulsions for the encapsulation and delivery of bioactive compounds in cosmetic or food formulations.

\section{Acknowledgments}

This work was supported by Associate Laboratory LSRE-LCM - UID / EQU / 50020/2019 - funded by national funds through FCT / MCTES (PIDDAC), and Foundation for Science and Technology (FCT, Portugal); and CIMO (UID/AGR/00690/2019) through FEDER under Program PT2020. The authors acknowledge the technical support provided by the i3s, University of Porto. Asma Sharkawy gratefully acknowledges financial support from the FCT doctoral grant (PD/BD/135085/2017).

\section{References}

Ali, B. H., Ziada, A., \& Blunden, G. (2009). Biological effects of gum arabic: A review of some recent research. Food and Chemical Toxicology, 47(1), 1-8. https://doi.org/10. 1016/J.FCT.2008.07.001.

Asfour, M. H., Elmotasem, H., Mostafa, D. M., \& Salama, A. A. A. (2017). Chitosan based Pickering emulsion as a promising approach for topical application of rutin in a solubilized form intended for wound healing: In vitro and in vivo study. International Journal of Pharmaceutics, 534(1-2), 325-338. https://doi.org/10.1016/J.IJPHARM. 
2017.10.044

Atarian, M., Rajaei, A., Tabatabaei, M., Mohsenifar, A., \& Bodaghi, H. (2019) Formulation of Pickering sunflower oil-in-water emulsion stabilized by chitosanstearic acid nanogel and studying its oxidative stability. Carbohydrate Polymers, 210, 47-55. https://doi.org/10.1016/J.CARBPOL.2019.01.008.

Avadi, M. R., Sadeghi, A. M. M., Mohammadpour, N., Abedin, S., Atyabi, F., Dinarvand, R., et al. (2010). Preparation and characterization of insulin nanoparticles using chitosan and Arabic gum with ionic gelation method. Nanomedicine Nanotechnology Biology and Medicine, 6(1), 58-63. https://doi.org/10.1016/J.NANO.2009.04.007.

Binks, B. P., \& Clint, J. H. (2002). Solid wettability from surface energy components: Relevance to pickering emulsions. Langmuir, 18(4), 1270-1273. https://doi.org/10. 1021/la011420k.

Butstraen, C., \& Salaün, F. (2014). Preparation of microcapsules by complex coacervation of gum Arabic and chitosan. Carbohydrate Polymers, 99, 608-616. https://doi.org/10. 1016/J.CARBPOL.2013.09.006.

Chevalier, Y., \& Bolzinger, M.-A. (2013). Emulsions stabilized with solid nanoparticles: Pickering emulsions. Colloids and Surfaces A, Physicochemical and Engineering Aspects, 439, 23-34. https://doi.org/10.1016/J.COLSURFA.2013.02.054.

Dai, L., Sun, C., Wei, Y., Mao, L., \& Gao, Y. (2018). Characterization of Pickering emulsion gels stabilized by zein/gum arabic complex colloidal nanoparticles. Food Hydrocolloids, 74, 239-248. https://doi.org/10.1016/J.FOODHYD.2017.07.040.

Dai, L., Zhan, X., Wei, Y., Sun, C., Mao, L., McClements, D. J., et al. (2018). Composite zein - propylene glycol alginate particles prepared using solvent evaporation: Characterization and application as Pickering emulsion stabilizers. Food Hydrocolloids, 85, 281-290. https://doi.org/10.1016/J.FOODHYD.2018.07.013.

Dickinson, E. (2003). Hydrocolloids at interfaces and the influence on the properties of dispersed systems. Food Hydrocolloids, 17(1), 25-39. https://doi.org/10.1016/S0268005X(01)00120-5.

Dickinson, E. (2017). Biopolymer-based particles as stabilizing agents for emulsions and foams. Food Hydrocolloids, 68, 219-231. https://doi.org/10.1016/J.FOODHYD.2016. 06.024.

Elsabee, M. Z., Morsi, R. E., \& Al-Sabagh, A. M. (2009). Surface active properties of chitosan and its derivatives. Colloids and Surfaces B, Biointerfaces, 74(1), 1-16. https://doi.org/10.1016/J.COLSURFB.2009.06.021.

Errezma, M., Mabrouk, A. B., Magnin, A., Dufresne, A., \& Boufi, S. (2018). Surfactant-free emulsion Pickering polymerization stabilized by aldehyde-functionalized cellulose nanocrystals. Carbohydrate Polymers, 202, 621-630. https://doi.org/10.1016/J. CARBPOL. 2018.09.018.

Harman, C. L. G., Patel, M. A., Guldin, S., \& Davies, G.-L. (2019). Recent developments in Pickering emulsions for biomedical applications. Current Opinion in Colloid \& Interface Science, 39, 173-189. https://doi.org/10.1016/J.COCIS.2019.01.017.

Hu, B., Han, L., Kong, H., Nishinari, K., Phillips, G. O., Yang, J., et al. (2019). Preparation and emulsifying properties of trace elements fortified gum arabic. Food Hydrocolloids, 88, 43-49. https://doi.org/10.1016/J.FOODHYD.2018.09.027.

Joye, I. J., \& McClements, D. J. (2014). Biopolymer-based nanoparticles and microparticles: Fabrication, characterization, and application. Current Opinion in Colloid \& Interface Science, 19(5), 417-427. https://doi.org/10.1016/J.COCIS.2014.07.002.

Lam, S., Velikov, K. P., \& Velev, O. D. (2014). Pickering stabilization of foams and emulsions with particles of biological origin. Current Opinion in Colloid \& Interface Science, 19(5), 490-500. https://doi.org/10.1016/J.COCIS.2014.07.003.

Larbi, F., García, A., del Valle, L. J., Hamou, A., Puiggalí, J., Belgacem, N., et al. (2018). Comparison of nanocrystals and nanofibers produced from shrimp shell $\alpha$-chitin: From energy production to material cytotoxicity and Pickering emulsion properties.
Carbohydrate Polymers, 196, 385-397. https://doi.org/10.1016/J.CARBPOL.2018.04. 094.

Laredj-Bourezg, F., Bolzinger, M.-A., Pelletier, J., \& Chevalier, Y. (2017). Pickering emulsions stabilized by biodegradable block copolymer micelles for controlled topical drug delivery. International Journal of Pharmaceutics, 531(1), 134-142. https:// doi.org/10.1016/J.JJPHARM.2017.08.065.

Li, J., Xu, X., Chen, Z., Wang, T., Lu, Z., Hu, W., et al. (2018). Zein/gum Arabic nanoparticle-stabilized Pickering emulsion with thymol as an antibacterial delivery system. Carbohydrate Polymers, 200, 416-426. https://doi.org/10.1016/J.CARBPOL. 2018.08.025.

Liu, W., Gao, H., McClements, D. J., Zhou, L., Wu, J., \& Zou, L. (2019). Stability, rheology, and $\beta$-carotene bioaccessibility of high internal phase emulsion gels. Food Hydrocolloids, 88, 210-217. https://doi.org/10.1016/J.FOODHYD.2018.10.012.

Marto, J., Gouveia, L., Duarte, A., Ribeiro, H., Simões, S., Gonçalves, L., et al. (2019). Starch-based pickering emulsions as platforms for topical antibiotic delivery: In vitro and in vivo studies. Polymers, 11(1), 108. https://doi.org/10.3390/polym11010108.

McClements, D. J., \& Gumus, C. E. (2016). Natural emulsifiers - Biosurfactants, phospholipids, biopolymers, and colloidal particles: Molecular and physicochemical basis of functional performance. Advances in Colloid and Interface Science, 234, 3-26. https://doi.org/10.1016/J.CIS. 2016.03.002.

Muxika, A., Etxabide, A., Uranga, J., Guerrero, P., \& de la Caba, K. (2017). Chitosan as a bioactive polymer: Processing, properties and applications. International Journal of Biological Macromolecules, 105, 1358-1368. https://doi.org/10.1016/J.IJBIOMAC. 2017.07.087.

Mwangi, W. W., Ho, K.-W., Tey, B.-T., \& Chan, E.-S. (2016). Effects of environmental factors on the physical stability of pickering-emulsions stabilized by chitosan particles. Food Hydrocolloids, 60, 543-550. https://doi.org/10.1016/J.FOODHYD.2016. 04.023.

Perrin, E., Bizot, H., Cathala, B., \& Capron, I. (2014). Chitin nanocrystals for pickering high internal phase emulsions. Biomacromolecules, 15(10), 3766-3771. https://doi. org/10.1021/bm5010417.

Pickering, S. U. (1907). Emulsions. Journal of the Chemical Society, 91, 2001-2021.

Tan, C., Xie, J., Zhang, X., Cai, J., \& Xia, S. (2016). Polysaccharide-based nanoparticles by chitosan and gum arabic polyelectrolyte complexation as carriers for curcumin. Food Hydrocolloids, 57, 236-245. https://doi.org/10.1016/J.FOODHYD.2016.01.021.

Wang, L.-J., Yin, S.-W., Wu, L.-Y., Qi, J.-R., Guo, J., \& Yang, X.-Q. (2016). Fabrication and characterization of Pickering emulsions and oil gels stabilized by highly charged zein/chitosan complex particles (ZCCPs). Food Chemistry, 213, 462-469. https://doi org/10.1016/J.FOODCHEM.2016.06.119.

Wei, Z., \& Huang, Q. (2019). Edible Pickering emulsions stabilized by ovotransferrin-gum arabic particles. Food Hydrocolloids, 89, 590-601. https://doi.org/10.1016/J. FOODHYD.2018.11.037.

Xiao, J., Li, Y., \& Huang, Q. (2016). Recent advances on food-grade particles stabilized Pickering emulsions: Fabrication, characterization and research trends. Trends in Food Science \& Technology, 55, 48-60. https://doi.org/10.1016/J.TIFS.2016.05.010.

Xiao, J., Wang, X., Perez Gonzalez, A. J., \& Huang, Q. (2016). Kafirin nanoparticlesstabilized Pickering emulsions: Microstructure and rheological behavior. Food Hydrocolloids, 54, 30-39. https://doi.org/10.1016/J.FOODHYD.2015.09.008.

Yang, J., Liu, L., \& Han, S. (2017). Preparation of octyl-grafted alginate-amide gel particle and its application in Pickering emulsion. Colloids and Surfaces A, Physicochemical and Engineering Aspects, 529, 320-327. https://doi.org/10.1016/J.COLSURFA.2017.06. 018. 